\title{
ANP32A modulates cell growth by regulating p38 and Akt activity in colorectal cancer
}

\author{
WEI YAN $^{1^{*}}$, ZHUN BAI $^{2 *}$, JUAN WANG $^{1,3}$, XUMEI LI $^{1}$, BIXIA CHI $^{4}$ and XU CHEN ${ }^{1}$ \\ ${ }^{1}$ College of Pharmacy, Guilin Medical University, Guilin 541004; ${ }^{2}$ Intensive Care Unit, \\ The Affiliated Zhuzhou Hospital XiangYa Medical College CSU, Zhuzhou, Hunan 412007; \\ ${ }^{3}$ Xiangya Hospital, Central South University, Changsha, Hunan 410008; ${ }^{4}$ Department of Gastroenterology, \\ The First People's Hospital of Yueyang, Yueyang, Hunan 414000, P.R. China
}

Received February 24, 2017; Accepted July 3, 2017

DOI: $10.3892 /$ or.2017.5845

\begin{abstract}
Acidic leucine-rich nuclear phosphoprotein-32A (ANP32A) possesses multiple biochemical activities, has been found to be decreased or absent in malignant tumors. However, new findings have shown that it is expressed in greater amounts in advanced cancers than in early-stage tumors. The role and clinical significance of ANP32A in colorectal cancer (CRC) is still unknown. In the present study, the expression of ANP32A was assessed in 68 CRC patients by IHC, and then the correlation of its expression with clinicopathological factors was investigated using the Allred, Klein and immune response scoring system analysis. Western blot and real-time PCR analyses were used to assess ANP32A expression and the activity of Akt and p38 in cancer and normal tissues. These data indicated a significant association between ANP32A expression and the activity of Akt and p38, besides the tumor differentiation status in CRC patients. IHC and western blotting data revealed that ANP32A was overexpressed in CRC patients, and ANP32A levels were higher in poorly differentiated tumors. Protein and mRNA analysis revealed that with a high expression of ANP32A, the activation of Akt was enhanced, while the p-38 phosphorylation level was decreased in CRC tissues. MTT assay and functional studies revealed that knockdown of ANP32A inhibited cell growth and induced p38 phosphorylation and Akt dephosphorylation. The present study indicated that ANP32A promoted CRC proliferation by inhibition of $\mathrm{p} 38$ and activation of Akt signaling pathways and suggested that ANP32A may play a potential role in CRC diagnosis and therapy.
\end{abstract}

Correspondence to: Dr Xu Chen, College of Pharmacy, Guilin Medical University, 109 Huancheng North 2 Road, Guilin 541004, P.R. China

E-mail: chenxu@glmc.edu.cn; junxuxu@163.com

${ }^{*}$ Contributed equally

Key words: ANP32A, p38, Akt, phosphorylation, cell growth, colorectal cancer

\section{Introduction}

Colorectal cancer (CRC) has one of the greatest incidences and mortality rates of any type of cancer in Europe and the United States. In China, the mortality of CRC ranks fifth in malignant tumors (1), but a rapid rise has been observed in recent years (2). Nearly $70 \%$ of CRC patients survive no more than 5 years after diagnosis. Surgical excision combined with chemotherapy is the preferred treatment for early-stage CRC, and thus can lead to good prognosis and higher overall survival rates. However, in advanced cases of CRC with multi-organ metastasis, only one-tenth of CRC patients survive to the 5 -year mark (3). Fluorouracil and capecitabine are widely used chemotherapeutic drugs for CRC (4). However, their use is usually limited due to the drug resistance of cancer. Although targeted agents anti-VEGF and anti-EGFR have been introduced for treatment of CRC, the overall death rate in advanced CRC remains high since EGFR therapeutic antibodies are only effective in $25 \%$ of advanced CRC patients (5). EGFR-targeted therapy fails mainly due to mutations in KRAS, NRAS and BRAF. Furthermore, amplification of ERBB2 and MET caused primary resistance $(5,6)$. Cells that acquired resistance to cetuximab remained sensitive to the combination of anti-EGFR and anti-MEK (6). Thus, early identification of molecular heterogeneity is crucial for the treatment of metastatic CRC.

ANP32A, known as PP32, I-1 ${ }^{\text {PP2A }}$, Lanp and PHAPI, is an acidic phosphate protein consisting of a leucine-rich repeat (LRR) amino terminal, and stretched acid-rich amino acids at the carboxy terminus (7), expressed in normal tissues as well as in pancreatic, breast and prostate cancer and other malignant tumors (8-10). ANP32A plays an important role in cell proliferation, apoptosis, transcriptional regulation, signal transduction and other processes (11). Most previous studies had shown that ANP32A is a tumor suppressor $(9,12-14)$, while recent studies found that ANP32A is highly expressed in hepatocellular carcinoma (15), and oral squamous cell carcinoma (OSCC) (16). Velmurugan et al reported that ANP32A is a potential prognostic factor and closely related to the survival rate of OSCC patients (16). In CRC, however, the roles and mechanisms of ANP32A are still not clear.

Acidic leucine-rich nuclear phosphoprotein-32A (ANP32A) has been identified as an inhibitor of protein 
phosphatase 2A (PP2A) (17), which is a serine/threonine protein phosphatase expressed in most mammalian tissues. PP2A has been shown to be a critical cellular regulator of cell metabolism, transcription, translation, RNA splicing, DNA replication, cell cycle progression, transformation and apoptosis (18). Studies have shown that PP2A activation induces apoptosis via the p38 MAPK-mediated pathway (18-22). The p38 MAP kinase is thought to be a promising cancer drug target, but its real therapeutic effect is unsatisfactory in CRC treatment (23). Zhang et al reported opposite responses in two subgroups of CRC cells to p38 inhibitors, which can explain why p38 inhibitors showed no positive effects on CRC in clinical settings (23). These findings are consistent with those reporting that p38 signaling has a dual function in colorectal tumorigenesis (24), and that the role and function of p38 in tumorigenesis may be affected by other unpredictable factors (25) including PP2A (23). Akt signaling has also been shown to be upregulated to promote colon cell growth required for the activity of PP2A (26). Numerous studies have revealed the relationship between ANP32A and PP2A, but there are few studies that have focused on the correlation between $\mathrm{p} 38$, Akt and ANP32A, which possesses the opposite effect of PP2A.

In the present study, we collected clinical specimens, analyzed the expression of ANP32A in CRC patients and its relationship with the differentiation of CRC. Meanwhile, due to the important role of $\mathrm{p} 38$ and Akt in CRCs, the relationship between ANP32A and these two proteins was investigated. The clarification of these results may be helpful in the treatment of CRC.

\section{Material and methods}

Clinical specimens. A total of 68 cases of CRC patients and matched tumor adjacent tissues were obtained from the Affiliated Hospital of Guilin Medical College to evaluate the relationship between the expression levels of ANP32A and the clinical/pathological factors. Tumor tissues were classified according to cancer tumor-node-metastasis (TNM) classification system issued by the Union for International Cancer Control. In order to avoid any factors that may affect the results of the experiment, all the selected specimens were examined without any treatment. All samples were studied with the consent of the patient and approval of the Guilin Medical College Ethics Committee.

CRC cell line. The CRC cell lines LoVo and SW480 were obtained from Xiangya Medical College of Central South University and stored in our laboratory. Cells were cultured in Dulbecco's modified Eagle's medium (DMEM) (HyClone, Shanghai, China) containing 10\% fetal bovine serum (FBS) (Gemini Biological Products, Calabasas, CA, USA) and $100 \mathrm{U} / \mathrm{ml}$ penicillin and streptomycin (Solarbio, Beijing, China) at $37^{\circ} \mathrm{C}$ in humidified atmosphere with $5 \% \mathrm{CO}_{2}$.

Immunohistochemistry (IHC) analysis. Tissue sections were fixed by formalin and embedded by paraffin in this experiment. The cancer tissues were cut into $4-\mu \mathrm{m}$ sections, and then hematoxylin/eosin staining was performed to confirm the presence of the original cancer as shown by its morphology. Tissue sections were dewaxed using xylene, and then tissue blocks were dehydrated in an ascending series of ethanol solutions. A $0.3 \%$ solution of hydrogen peroxide in methanol was added for $10 \mathrm{~min}$ to eliminate the effects of endogenous peroxidase and incubated with $10 \mathrm{mmol} / \mathrm{l}$ citrate buffer $(\mathrm{pH} 6.0)$. The tissues were blocked at $37^{\circ} \mathrm{C}$ with $5 \%$ goat serum (Solarbio). The sections were then incubated with ANP32A rabbit polyclonal antibody (Abcam, Cambridge, UK) in room temperature for $1 \mathrm{~h}$. The secondary antibody used was a horseradish peroxidase-conjugated antibody (MXB, Fuzhou, China). Each tumor was scored based on the ratio of the intensity of the nucleus and cytoplasm: negative staining and weak positive staining, \pm ; moderate staining, $1+$; and strong staining, $2+$. Staining intensity was confirmed by two pathologists. For the Allred scoring system, cut-off scores $\leq 4$ and $>4$ were defined as low and high expression levels, respectively.

Cell transfection. Double-stranded small interfering RNA (siRNA) oligonucleotides were obtained from Bioligo, (Shanghai, China). The siRNA sequence targeting ANP32A (si-ANP32A) was 5-CAAUCGCAAACUUACCAAAGT-3; a fluorescently-labeled non-specific pri-miR sequence was used as a negative control (sequence targeting was 5-UUCUCCGAA CGUGUCACGUTT-3).

Total RNA isolation and quantitative real-time PCR. Total RNA was isolated from SW480 cells transfected with si-ANP32A and CRC tissue using TRIzol reagent (Tiangen Biotech Co., Ltd., Beijing, China). The isolated RNA was used as a template for the reverse transcription reaction. The primers were designed according to the corresponding cDNA sequences in GenBank. These primers listed in Table I were designed to detect ANP32A, AKT, p53 and $\beta$-actin (positive control).

Western blotting. Total protein was extracted from SW480 and LoVo cells, and CRC tissues. The primary antibodies of ANP32A (Abcam), p-Akt, p38, and p-p38 (Wanleibio, Shenyang, China) were used to detect the protein expression. $\beta$-actin (ZSGB-BIO, Beijing, China) was used as a loading control. Proteins of different molecular weights were isolated using 12\% SDS-PAGE. A nitrocellulose membrane was then used to transfer the target protein. The membrane was incubated in $5 \%$ skim milk overnight at $4^{\circ} \mathrm{C}$, and then treated with horseradish peroxidase-labeled anti-mouse or anti-rabbit secondary antibodies for $1 \mathrm{~h}$ at $37^{\circ} \mathrm{C}$ and detected with an enhanced chemiluminescence (ECL) reagent (Bio-Rad, Hercules, CA, USA).

MTT analysis. SW480 cells were divided into a si-ANP32A, negative and blank control group. Each group had quadruplicate wells for treatment, respectively. Cells at $2 \times 10^{4}$ cells $/ \mathrm{ml}$ were seeded into 96-well plates at a final volume of $100 \mu 1$ and incubated at $37^{\circ} \mathrm{C}$ and $5 \% \mathrm{CO}_{2}$ in saturated humidity. Cells were incubated with $20 \mu \mathrm{l}$ of MTT for $4 \mathrm{~h}$ at $37^{\circ} \mathrm{C}$ and $5 \% \mathrm{CO}_{2}$ in saturated humidity and treated with $150 \mu \mathrm{l}$ of dimethyl sulfoxide (DMSO) after removal of the supernatant, and then shaken for $10 \mathrm{~min}$ on a plate shaker. Dissolved the optical density (OD) value of each well was measured at $490 \mathrm{~nm}$ wavelength by TECAN M200 (Tecan Group Ltd., 
Table I. Sequences of primers used in quantitative real-time PCR.

\begin{tabular}{lll}
$\begin{array}{l}\text { RNA } \\
\text { species }\end{array}$ & \multicolumn{1}{c}{ Primer pairs } \\
\hline ANP32A & $\begin{array}{l}\text { Sense } \\
\text { Antisense }\end{array}$ & $\begin{array}{l}\text { 5'-CACCTCAATCGCAAACTTACCA-3' } \\
\text { 5-AACACATTTTCTCGGTAGTCGTT-3' }\end{array}$ \\
& $\begin{array}{l}\text { Sense } \\
\text { Antisense }\end{array}$ & 5'-AAAGACCTGTACGCCAACAC-3' \\
Akt & Sense & 5'-AGAACCTCACTCCTGCTTGCTGAT-3' \\
& Antisense & 5'-CTCATGGTCCTGGTTGTAGA-3' \\
p53 & Sense & 5'-TCAACAAGATGTTTTGCCAACTG-3' \\
& Antisense & 5-ATGTGCTGTGACTGCTTGTAGATG-3
\end{tabular}

ANP32A, acidic leucine-rich nuclear phosphoprotein-32A.

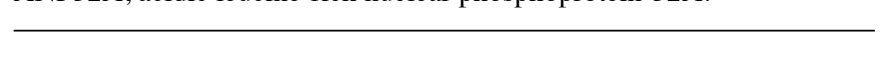

Männedorf, Switzerland). All experiments were performed at least three times. Growth inhibition rate $=($ control group OD value - OD value of experimental group) / control group OD values x $100 \%$.

Statistical analysis. All data in the present study were evaluated using SPSS version 18.0 software (SPSS, Inc., Chicago, IL, USA). The statistical significance of the data was evaluated using the Student's t-test. All data are expressed as mean \pm SD. The relationship between the expression of ANP32A and the clinicopathological parameters of CRC was assessed by the $\chi^{2}$ test. For all tests, the level of significance was set at $\mathrm{P}<0.05$.

\section{Results}

ANP32A expression in tumor and normal tissues of CRC patients. The clinical data of 68 patients with CRC are summarized in Table II. According to the histological grade of $\mathrm{CRC}, 23$ patients well-differentiated tumors, whereas the remaining 45 patients had moderately or poorly differentiated tumors. In order to investigate the expression of ANP32A in CRC, we performed IHC. As shown in Fig. 1, positive IHC staining was mainly localized in the cytoplasm and nucleus of CRC cells, and ANP32A was highly expressed in poorly differentiated CRC. Total protein was extracted from CRC and matched tumor adjacent tissues, then subjected to western blot analysis was used to detect ANP32A expression. The results revealed that the expression of ANP32A was higher in CRC, which was consistent with the aforementioned IHC data in which a high level of ANP32A expression was detected. In addition, high ANP32A protein expression was found to have a statistically significant relationship to tumor differentiation $(\mathrm{P}=0.021)$ (Table III).

Cell proliferation is detected by MTT assay. In order to investigate the effect of ANP32A on cell proliferation, the cell viability was assessed in the present study via silencing of ANP32A in SW480 cells. The MTT assay revealed that the growth rate of SW480 cells transfected with siRNA targeting ANP32A was lower than that of the negative or blank control groups $(\mathrm{P}<0.05)$. The results revealed that the growth
Table II. Demographics and characteristics among CRC patients.

\begin{tabular}{|c|c|c|}
\hline Factors & No. & $\%$ \\
\hline \multicolumn{3}{|l|}{ Sex } \\
\hline Female & 43 & 63.24 \\
\hline Male & 25 & 36.76 \\
\hline \multicolumn{3}{|l|}{ Age, years } \\
\hline$\leq 49$ & 12 & 17.65 \\
\hline $50-59$ & 22 & 32.35 \\
\hline $60-69$ & 23 & 33.82 \\
\hline$\geq 70$ & 11 & 16.18 \\
\hline \multicolumn{3}{|c|}{$\mathrm{T}$ (tumor size) } \\
\hline TIS & 3 & 4.41 \\
\hline I & 9 & 13.24 \\
\hline II & 19 & 27.94 \\
\hline III & 36 & 52.94 \\
\hline IV & 1 & 1.47 \\
\hline \multicolumn{3}{|c|}{ N (lymph node) } \\
\hline No & 48 & 70.59 \\
\hline N1 & 14 & 20.59 \\
\hline $\mathrm{N} 2$ & 6 & 8.82 \\
\hline \multicolumn{3}{|c|}{ M (metastasis) } \\
\hline M0 & 62 & 91.18 \\
\hline M1 & 6 & 8.82 \\
\hline \multicolumn{3}{|c|}{ AJCC cancer stage } \\
\hline 0 & 3 & 4.41 \\
\hline $\mathrm{I}$ & 25 & 36.76 \\
\hline \multicolumn{3}{|l|}{ II } \\
\hline IIA & 18 & 26.47 \\
\hline \multicolumn{3}{|l|}{ III } \\
\hline IIIA & 1 & 1.47 \\
\hline IIIB & 12 & 17.65 \\
\hline IIIC & 3 & 4.41 \\
\hline \multicolumn{3}{|l|}{ IV } \\
\hline IVA & 4 & 5.88 \\
\hline IVB & 2 & 2.94 \\
\hline \multicolumn{3}{|c|}{ Histological grade } \\
\hline Well & 23 & 33.82 \\
\hline Moderate & 42 & 61.76 \\
\hline Poor & 3 & 4.41 \\
\hline
\end{tabular}

CRC, colorectal cancer; AJCC, American Joint Committee on Cancer; TIS, tumor in situ.

inhibition rates of the siRNA treatment group were 8.4, 15.6 and $14.1 \%$ at 24,48 and $72 \mathrm{~h}$, respectively (Fig. 2B). There was no significant difference between the negative or blank control groups. Collectively, these results indicated that knockdown of ANP32A can prevent proliferation of SW480 cells effectively.

Silencing of ANP32A affects the expression of genes associated with cell proliferation in SW480 cells. The expression of 
A .
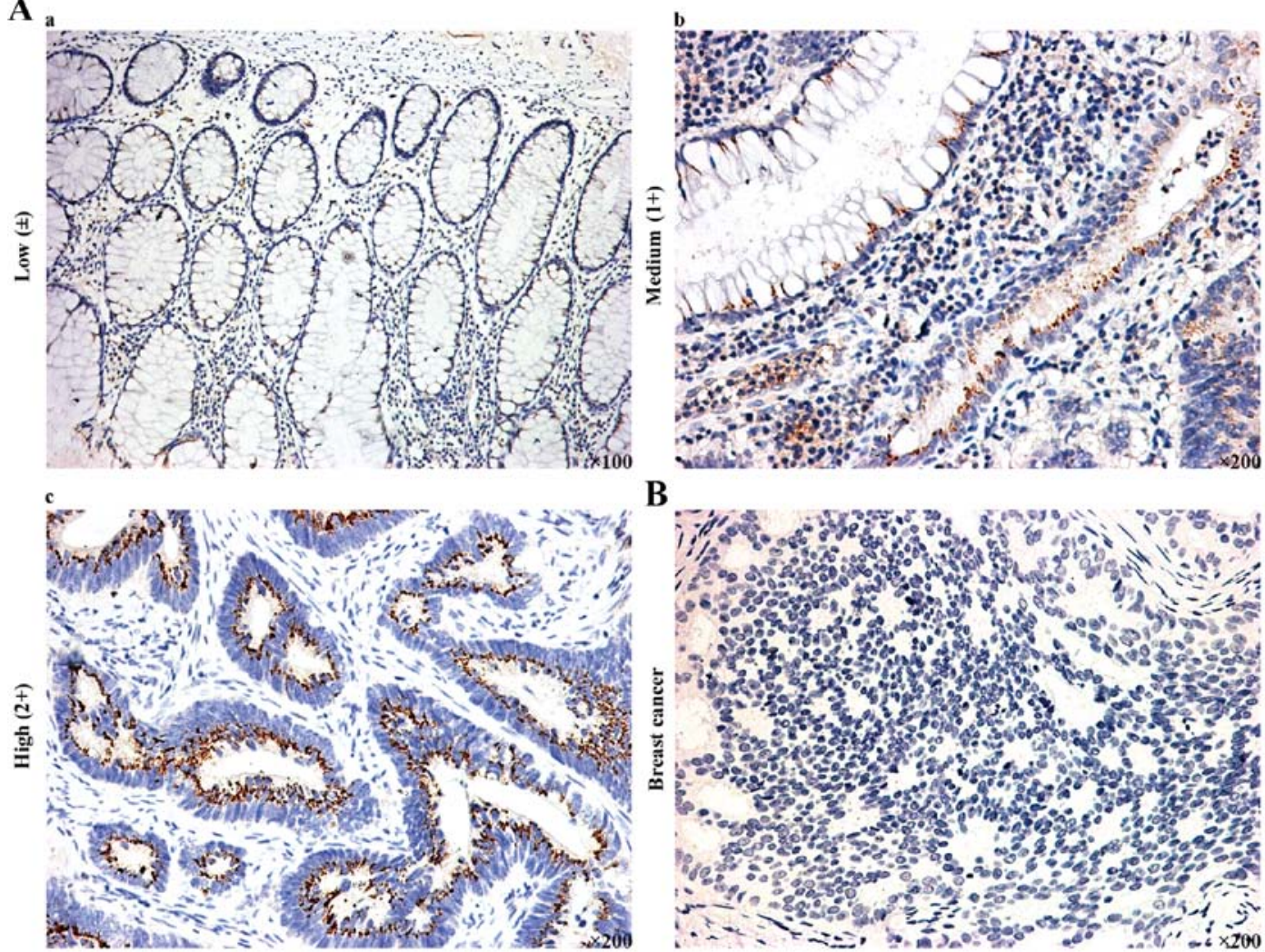

B

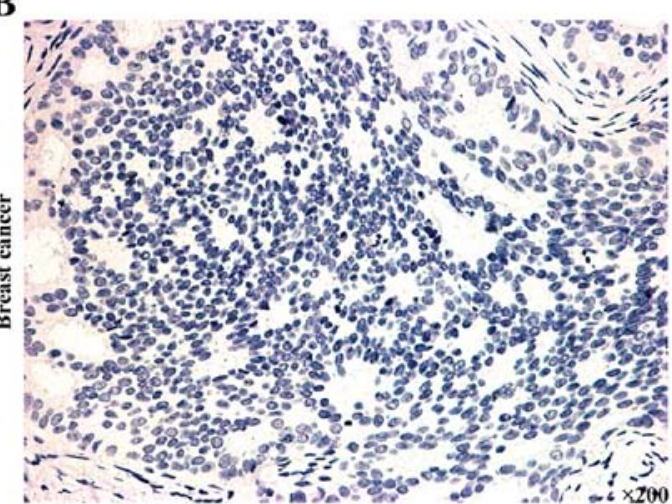

C

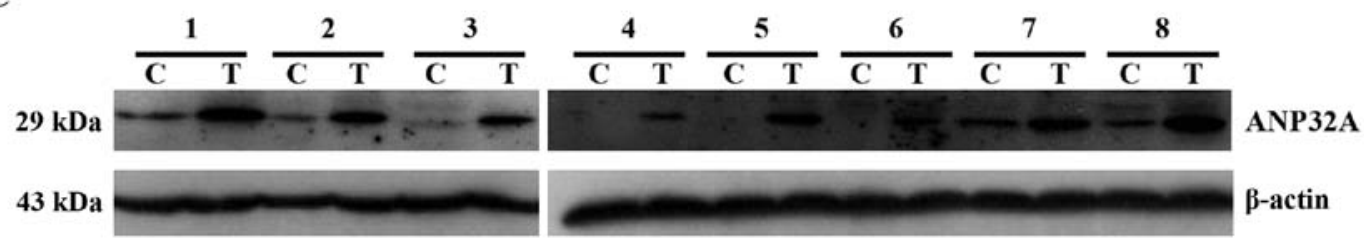

Figure 1. Higher expression of ANP32A in colorectal cancer (CRC) patients. (A) The staining intensities of ANP32A were identified in CRC tissues. Cells were stained using a scale $( \pm, 1+, 2+)$, classified as follows: representative of low ANP32A, medium ANP32A and high ANP32A staining. (B) Breast cancer tissues were used as a negative control. (C) The protein level of ANP32A was analyzed by immunoblotting in CRC patients, and $\beta$-actin served as an internal control. There were 8 CRC patients randomly selected, of which group $\mathrm{C}$ were tumor-adjacent tissues of CRC patients and group $\mathrm{T}$ were cancer tissues of CRC patients.

Table III. Correlation of ANP32A expression with clinicalpathological characteristics using the Allred scoring system among CRC patients.

\begin{tabular}{lcccc}
\hline & \multicolumn{2}{c}{ Allred } & & \\
\cline { 2 - 4 } Factors & $\begin{array}{c}\text { Low no. } \\
(\mathrm{n}=37)\end{array}$ & $\begin{array}{c}\text { High no. } \\
(\mathrm{n}=31)\end{array}$ & $\chi^{2}$ & P-value \\
\hline Histological grade & & & 5.328 & 0.021 \\
Well & 17 & 6 & & \\
Moderate/poor & 20 & 25 & & \\
\hline
\end{tabular}

CRC, colorectal cancer; ANP32A, acidic leucine-rich nuclear phosphoprotein-32A.

ANP32A was detected by western blotting. Higher levels of p-Akt expression were observed in CRC tissues than in normal tumor-adjacent tissues. Unlike the expression patterns of p-Akt, p38 and p-p38 were overexpressed in normal tumor-adjacent tissues (Fig. 3). As shown in Fig. 3A, the mRNA expression of total Akt was higher in the CRC tissues, as was the expression of ANP32A in the CRC tissues. The protein expression levels of ANP32A and p-Akt were higher in the CRC tissues and the level of phosphorylated p-38 was lower (Fig. 3B).

To elucidate the details underlying the molecular mechanism of ANP32A in CRC, SW480 cells with higher ANP32A expression were selected for the current investigation of the effects of ANP32A knockdown (Fig. 4A). For this reason, pre-treatment with siRNA targeting ANP32A for $48 \mathrm{~h}$, and then quantitative real-time PCR experiments were performed to determine whether knockdown of ANP32A subsequently regulates Akt transcription. The data revealed that Akt mRNA expression was decreased by the inhibition of the expression of ANP32A, whereas the levels of p53 were increased (Fig. 4B). When normalizing to $\beta$-actin, ANP32A mRNA expression in the CRC samples was nearly 8.09-fold higher than that in the matched non-cancerous tissues, and the data were consistent with the previous western blotting test in which high expression of ANP32A was detected (Fig. 4B). The results indicated 
A a

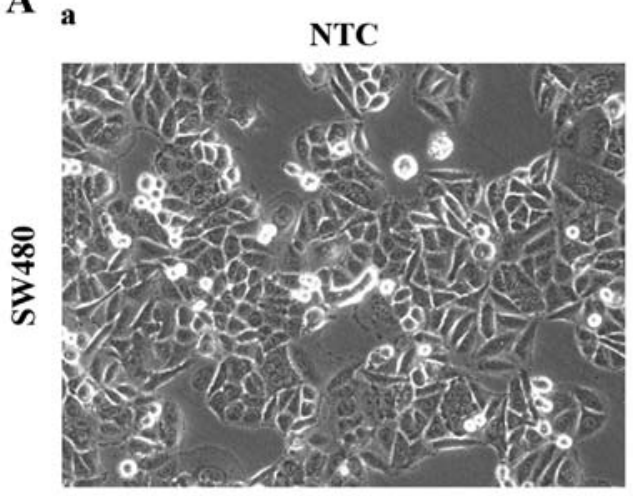

c

Si-ANP32A

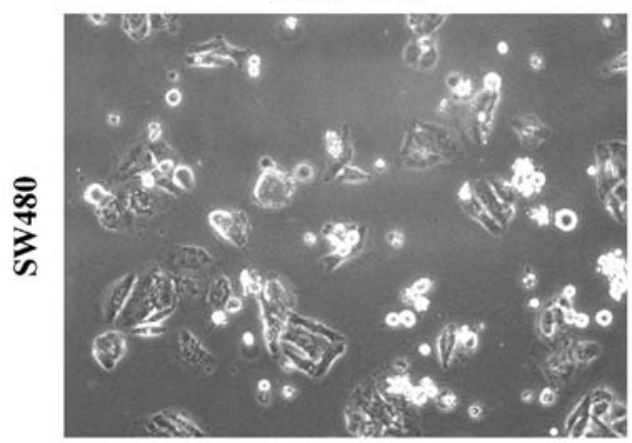

b

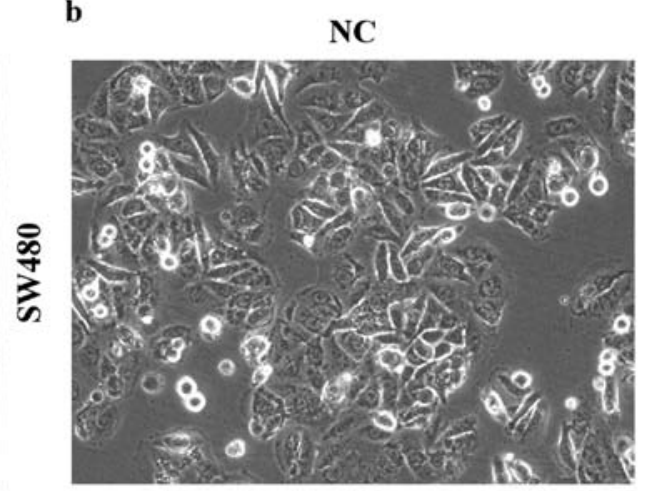

B

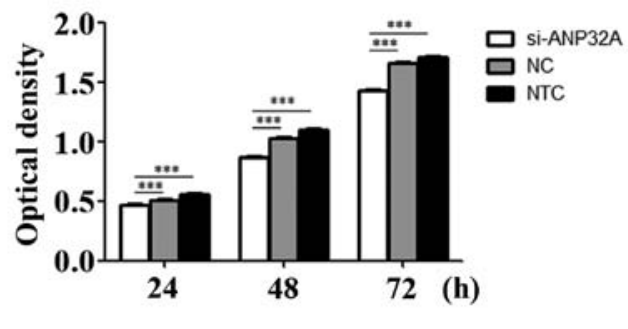

Figure 2. Knockdown of ANP32A by siRNA decreases the cell viability in SW480 cells. (A) SW480 cells were transfected with si-ANP32A or siControl for $24 \mathrm{~h}$ and microscopically photographed by inverted fluorescence microscope. (B) An MTT assay was used to detect the proliferation of SW480 cells after transfection at 24,48 and $72 \mathrm{~h}$, respectively. 'NC' refers to the negative control group, and 'NTC' to the blank control group. Densitometry values are represented in a bar chart, and the data is expressed as the mean values \pm SD. The asterisks indicate significant differences among the siControl and si-ANP32A and blank control group $\left({ }^{* * *} \mathrm{P}<0.001\right)$.

A

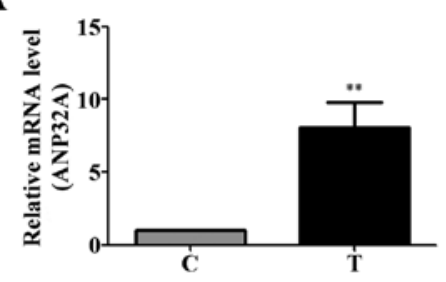

B

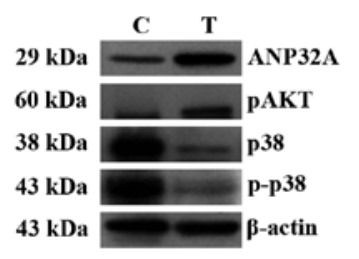

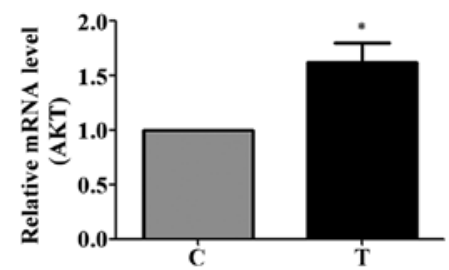
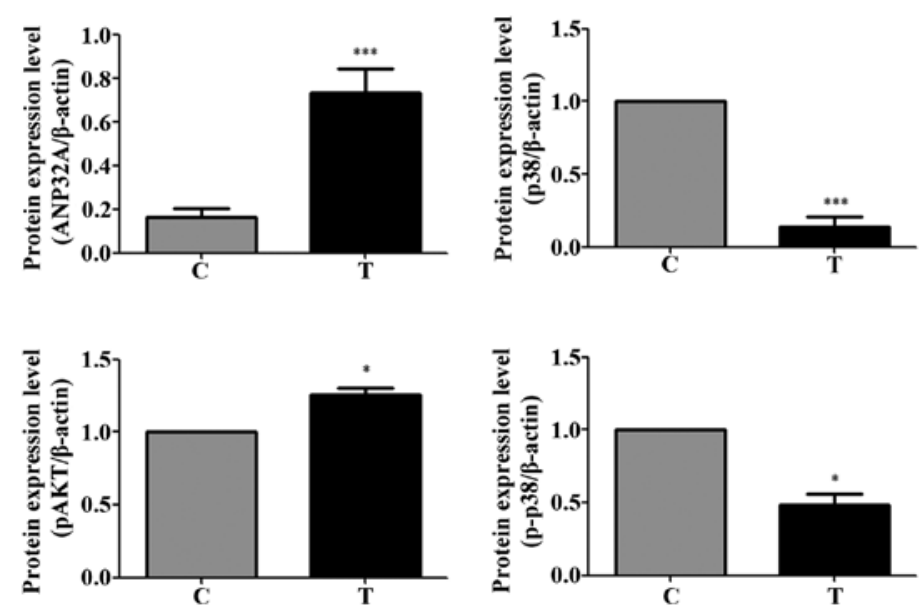

Figure 3. Expression of ANP32A, pAKT and p38 in cancer and tumor-adjacent tissues. (A) The expression of ANP32A or Akt mRNA was analyzed using quantitative RT-PCR and the results were calculated using $2^{-\Delta \Delta \mathrm{Ct}}$. (B) The expression of ANP32A, pAKT, p38 and p-p38 were detected using western blot analysis. $\beta$-actin was used as an internal control for each protein. ' $\mathrm{C}$ ' represents tumor-adjacent tissues of $\mathrm{CRC}$ patients, and ' $\mathrm{T}$ ' represents cancer tissues of CRC patients. Densitometry values are represented in bar charts. Data is expressed as the mean values \pm SD. The asterisks indicate significant differences between the ' $\mathrm{C}$ ' control and the ' $\mathrm{T}$ ' tumor group $\left({ }^{*} \mathrm{P}<0.05,{ }^{* * * *} \mathrm{P}<0.001\right)$. 
A

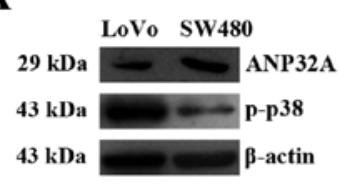

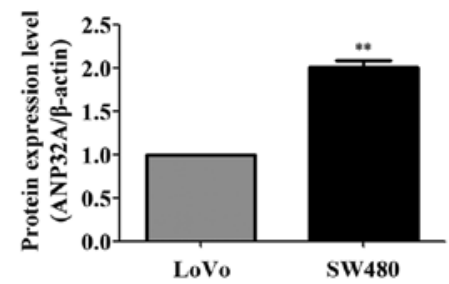

B

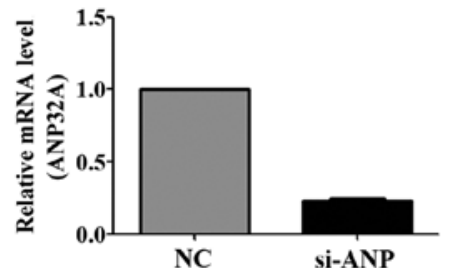

C

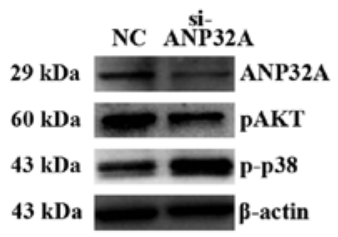

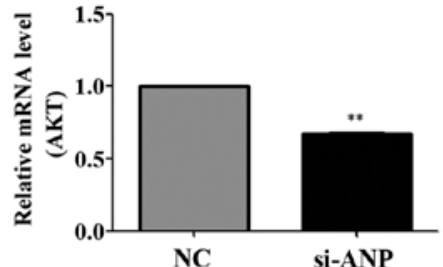
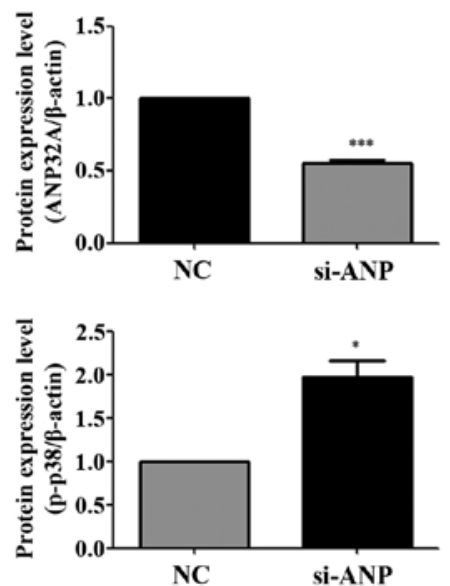
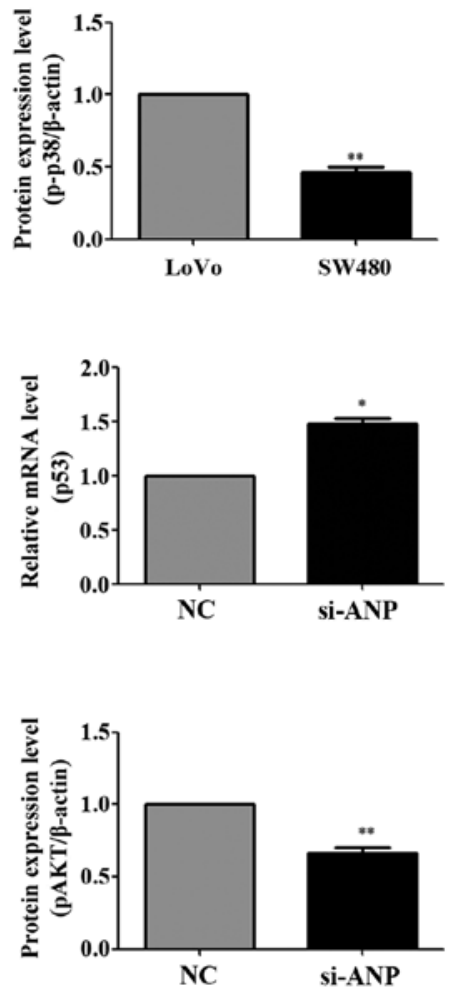

Figure 4. Effect of ANP32A on the expression of genes associated with proliferation. (A) The expression of ANP32A and p-p38 were detected using western blotting in SW480 and LoVo cells. (B) After SW480 cells were transfected with siControl (siCtrl) or si-ANP32A, the expression of ANP32A, Akt and p53 were analyzed by quantitative RT-PCR. (C) The expression of p-Akt and p-p38 were analyzed using western blotting after ANP32A expression knockdown. $\beta$-actin was used as a loading control. Densitometry values are represented in bar charts, the data is expressed as the mean values \pm SD. The asterisks indicate the significant differences between LoVo and SW480 cells and the differences between the siControl and si-ANP32A groups $\left({ }^{* *} \mathrm{P}<0.05,{ }^{* *} \mathrm{P}<0.01,{ }^{* * *} \mathrm{P}<0.001\right)$.

that knockdown of ANP32A resulted in the decrease of p-Akt expression, but the increase of the expression of p-p38 (Fig. 4C). All of these data strongly demonstrated that silencing of ANP32A inhibited the Akt activation and increased the expression of p-p38 in SW480 cells. These results demonstrated that the expression of ANP32A is associated with the level of Akt and that it may promote the proliferation of CRC cells.

\section{Discussion}

Colorectal cancer (CRC) is a high-incidence and high-mortality form of cancer (27). The findings of numerous different studies have shown that several factors induce the formation of CRC, included genetic and epigenetic alterations $(28,29)$. Some of these gene alterations can be applied as biomarkers for cancer diagnosis and provide strategies for cancer therapy (30). The human ANP32A gene is expressed differently in different tumor tissues. Low expression has been reported in prostate $(31)$, pancreatic $(9,32)$ and breast cancer $(33,34)$, and B cell lymphoma (13). Early studies have shown that ANP32A is expressed as a modulator of cancer cell apoptosis in vitro and in vivo $(35,36)$, including human non-small cell lung cancer (NSCLC) (37). In pancreatic cancer, Brody et al demonstrated that considerable expression of ANP32A was found in well-differentiated tumors, however decreased or absent expression of ANP32A was found to be related to poor differentiation (38). Notably, Velmurugan et al found ANP32A levels to be higher in tumor tissues and that high levels of ANP32A expression were associated with poor tumor differentiation in OSCC (16). Shi et al found that ANP32A was overexpressed in CRC using laser capture microdissection (LCM) and two-dimensional difference gel electrophoresis (2D-DIGE) (39), but no further information is available concerning ANP32A expression with tumor differentiation or its significance to the development of CRC. In the present study, the expression of ANP32A was evaluated in CRC and adjacent tumor-free tissue samples. IHC, western blotting, and real-time PCR analysis demonstrated that CRC specimens exhibited significantly higher ANP32A protein and mRNA levels than normal tissues nearby. There is a statistically positive correlation between the ANP32A protein expression levels and the degrees of tumor differentiation in cancer tissues. Overexpression of ANP32A has been shown to 
suppress tumor cell growth and induce apoptosis in breast and non-small-cell lung cancer $(33,37)$. It has been reported that artificially introducing ANP32A expression into pancreatic cell lines in which it has been absent can increase the rate of G1 arrest relative to control cells (38). The present study demonstrated that ANP32A knockdown by RNA interference suppresses the proliferation of CRC cells. These results revealed that ANP32A promotes tumor development in CRC cells by accelerating cell proliferation and that overexpression of ANP32A has an important role in tumorigenesis and progression. These results were inconsistent with results associated with other tumors. The results indicated that the expression of ANP32A varies in different types of cancer, and may have the opposite effects in different tumor tissues.

To identify the possible mechanisms by which ANP32A enhances the survival of colon cancer cells, the effects of ANP32A on molecules involved in the proliferation of CRC were investigated. Numerous studies have shown that p38 has a promotive effect in CRC, but newer studies have shown that p38 MAPK can act as a tumor suppressor in CRC (40), and that it constitutes a potential molecular target in the inhibition of colorectal carcinogenesis $(41,42)$. A previous study revealed that the activation of $\mathrm{p} 38$ can lead to an increase in apoptosis in the LoVo CRC cell line (43). The p38 inhibitor SB203580 can reverse the effect of apoptosis upon curcumol treatment in LoVo cells (43). PP2A, an inhibitor of ANP32A, is a negative regulator of $\mathrm{p} 38$ activation $(22,44-46)$. It can be easily hypothesized that ANP32A may have a positive effect in $\mathrm{p} 38$ regulation. Habrukowich et al revealed that knockdown of ANP32A expression induced p38 phosphorylation in human umbilical vein endothelial cells (HUVEC) (47). To confirm the activity of p38 in colon cancer tissues, the expression of phosphorylated p38 was assessed in CRC specimens and ANP32A silenced SW480 cells. Protein analysis data revealed the p38 phosphorylation levels to be lower in CRC than in normal cells, and that silencing of ANP32A upregulated p38 activation. Akt, according to previous studies, plays a key role in protein synthesis, cell metabolism and survival, and it is a key regulator of proliferation and metastasis in CRC cells (48-50). Phosphorylated Akt (p-Akt) can be used as a tissue biomarker to identify patients with favorable prognosis and to identify suitable therapeutic targets $(41,51)$. It has been demonstrated that in addition to activation of $\mathrm{p} 38$, the activity of PP2A was also significantly increased by triptolide and hydroxycamptothecin, and the Akt survival pathway was also inhibited in A549 cells (46). Van Kanegan et al found that PP2A inhibition potentiates Akt phosphorylation in PC12 cells (52). To further confirm the effect of ANP32A in CRC and determine the correlation between ANP32A and p-Akt, function analysis of ANP32A was performed in SW480 cells. These protein and mRNA results revealed that, as the expression of ANP32A increased in colorectal tissues, phosphorylated Akt was upregulated. The results also revealed there to be less expression of Akt in the ANP32A-silenced cells than in other cells at both the mRNA and protein levels.

In summary, ANP32A was overexpressed in CRC patients. Knockdown of ANP32A was found to inhibit CRC SW480 cell growth and the net effect of ANP32A silencing was hyperphosphorylation of p38 and dephosphorylation of Akt. These results demonstrated that ANP32A may be a suitable molecule for the development of CRC, and the mechanism by which ANP32A promotes colon cancer growth may involve p38 inactivation and Akt activation. Further studies are required to improve our understanding of the specific effect of ANP32A in cancer. The involvement of other factors and signaling pathways in ANP32A interaction remains to be explored.

\section{Acknowledgements}

The present study was supported by the National Natural Science Foundation of China (grant no. 31460229), the Natural Science Fund of Guangxi Province (grant no. 2015GXNSFAA139313), the Scientific Research and Technology Development Projects of Guilin (grant no. 20140105-8), and the Small Talent Highland Fund in Guangxi (grant nos. 1415 and 201707). The authors would like to thank Professor Junfei Jin for his valuable suggestions and editorial contributions.

\section{References}

1. Chen W, Zheng R, Zeng H, Zhang S and He J: Annual report on status of cancer in China, 2011. Chin J Cancer Res 27: 2-12, 2015.

2. Wang H, Chen Y and Wu G: SDHB deficiency promotes TGF $\beta$ mediated invasion and metastasis of colorectal cancer through transcriptional repression complex SNAIL1-SMAD3/4. Transl Oncol 9: 512-520, 2016.

3. Jin XQ, Liu P and Zhang QP:[Genetic susceptibility in children with incomplete Kawasaki disease. Zhongguo Dang Dai Er Ke Za Zhi 17: 663-667, 2015.

4. Segal NH and Saltz LB: Evolving treatment of advanced colon cancer. Annu Rev Med 60: 207-219, 2009.

5. Misale S, Di Nicolantonio F, Sartore-Bianchi A, Siena S and Bardelli A: Resistance to anti-EGFR therapy in colorectal cancer: From heterogeneity to convergent evolution. Cancer Discov 4: 1269-1280, 2014.

6. Misale S, Yaeger R, Hobor S, Scala E, Janakiraman M, Liska D, Valtorta E, Schiavo R, Buscarino M, Siravegna G, et al: Emergence of KRAS mutations and acquired resistance to anti-EGFR therapy in colorectal cancer. Nature 486: 532-536, 2012.

7. Opal P, Garcia JJ, McCall AE, Xu B, Weeber EJ, Sweatt JD, Orr HT and Zoghbi HY: Generation and characterization of LANP/pp32 null mice. Mol Cell Biol 24: 3140-3149, 2004.

8. Adegbola $\mathrm{O}$ and Pasternack GR: Phosphorylated retinoblastoma protein complexes with pp32 and inhibits pp32-mediated apoptosis. J Biol Chem 280: 15497-15502, 2005.

9. Williams TK, Costantino CL, Bildzukewicz NA, Richards NG, Rittenhouse DW, Einstein L, Cozzitorto JA, Keen JC, Dasgupta A, Gorospe M, et al: pp32 (ANP32A) expression inhibits pancreatic cancer cell growth and induces gemcitabine resistance by disrupting HuR binding to mRNAs. PLoS One 5: e15455, 2010.

10. Brody JR, Kadkol SS, Hauer MC, Rajaii F, Lee J and Pasternack GR: pp32 reduction induces differentiation of TSU-Pr1 cells. Am J Pathol 164: 273-283, 2004.

11. Wang S, Wang Y, Lu Q, Liu X, Wang F, Ma X, Cui C, Shi C, Li J and Zhang D: The expression and distributions of ANP32A in the developing brain. Biomed Res Int 2015: 207347, 2015.

12. Pan W, da Graca LS, Shao Y, Yin Q, Wu H and Jiang X: PHAPI/ pp32 suppresses tumorigenesis by stimulating apoptosis. J Biol Chem 284: 6946-6954, 2009.

13. Schramedei K, Mörbt N, Pfeifer G, Läuter J, Rosolowski M, Tomm JM, von Bergen M, Horn F and Brocke-Heidrich K: MicroRNA-21 targets tumor suppressor genes ANP32A and SMARCA4. Oncogene 30: 2975-2985, 2011.

14. Mazroui R, Di Marco S, Clair E, von Roretz C, Tenenbaum SA, Keene JD, Saleh M and Gallouzi IE: Caspase-mediated cleavage of $\mathrm{HuR}$ in the cytoplasm contributes to pp32/PHAP-I regulation of apoptosis. J Cell Biol 180: 113-127, 2008.

15. Li C, Ruan HQ, Liu YS, Xu MJ, Dai J, Sheng QH, Tan YX, Yao ZZ, Wang HY, Wu JR, et al: Quantitative proteomics reveal up-regulated protein expression of the SET complex associated with hepatocellular carcinoma. J Proteome Res 11: 871-885, 2012. 
16. Velmurugan BK, Yeh K-T, Lee C-H, Lin SH, Chin MC, Chiang SL, Wang ZH, Hua CH, Tsai MH, Chang JG, et al: Acidic leucine-rich nuclear phosphoprotein-32A (ANP32A) association with lymph node metastasis predicts poor survival in oral squamous cell carcinoma patients. Oncotarget 7: 10879-10890, 2016.

17. Li M, Makkinje A and Damuni Z: Molecular identification of $\mathrm{I}_{1}{ }^{\mathrm{PP} 2 \mathrm{~A}}$, a novel potent heat-stable inhibitor protein of protein phosphatase 2A. Biochemistry 35: 6998-7002, 1996.

18. Schönthal AH: Role of serine/threonine protein phosphatase $2 \mathrm{~A}$ in cancer. Cancer Lett 170: 1-13, 2001.

19. Grethe S and Pörn-Ares MI: p38 MAPK regulates phosphorylation of Bad via PP2A-dependent suppression of the MEK1/2-ERK1/2 survival pathway in TNF-alpha induced endothelial apoptosis. Cell Signal 18: 531-540, 2006.

20. Meng G, Sun Y, Fu W, Guo Z and Xu L: Microcystin-LR induces cytoskeleton system reorganization through hyperphosphorylation of tau and HSP27 via PP2A inhibition and subsequent activation of the p38 MAPK signaling pathway in neuroendocrine (PC12) cells. Toxicology 290: 218-229, 2011.

21. Garcia A, Cayla X, Guergnon J, Dessauge F, Hospital V, Rebollo MP, Fleischer A and Rebollo A: Serine/threonine protein phosphatases PP1 and PP2A are key players in apoptosis. Biochimie 85: 721-726, 2003.

22. Boudreau RT, Conrad DM and Hoskin DW: Apoptosis induced by protein phosphatase $2 \mathrm{~A}$ ( $\mathrm{PP} 2 \mathrm{~A}$ ) inhibition in $\mathrm{T}$ leukemia cells is negatively regulated by PP2A-associated p38 mitogenactivated protein kinase. Cell Signal 19: 139-151, 2007.

23. Shang C and Huang S: PP2A level in colorectal cancer cells predicts the response of p38 targeted therapy. EBioMedicine 2: $1848-1849,2015$

24. Gupta J, del Barco Barrantes I, Igea A, Sakellariou S, Pateras IS, Gorgoulis VG and Nebreda AR: Dual function of p38 $\alpha$ MAPK in colon cancer: Suppression of colitis-associated tumor initiation but requirement for cancer cell survival. Cancer Cell 25: 484-500, 2014.

25. Wagner EF and Nebreda AR: Signal integration by JNK and p38 MAPK pathways in cancer development. Nat Rev Cancer 9: 537-549, 2009

26. Kumar A, Pandurangan AK, Lu F, Fyrst H, Zhang M, Byun HS, Bittman R and Saba JD: Chemopreventive sphingadienes downregulate Wnt signaling via a PP2A/Akt/GSK3 $\beta$ pathway in colon cancer. Carcinogenesis 33: 1726-1735, 2012.

27. Smith RA, Andrews K, Brooks D, DeSantis CE, Fedewa SA, Lortet-Tieulent J, Manassaram-Baptiste D, Brawley OW and Wender RC: Cancer screening in the United States, 2016: A review of current American Cancer Society guidelines and current issues in cancer screening. CA Cancer J Clin 66: 96-114, 2016.

28. Okugawa Y, Grady WM and Goel A: Epigenetic alterations in colorectal cancer: Emerging biomarkers. Gastroenterology 149: 1204-1225.e12, 2015.

29. Bae JM, Kim JH and Kang GH: Epigenetic alterations in colorectal cancer: The $\mathrm{CpG}$ island methylator phenotype. Histol Histopathol 28: 585-595, 2013.

30. Weng KP, Hsieh KS, Huang SH, Wu HW, Chien JH, Lin CC, Tang CW, Ou SF, Huang SJ and Ger LP: Myeloperoxidase genetic polymorphisms and susceptibility to Kawasaki disease in Taiwanese children. J Microbiol Immunol Infect 49: 788-796, 2016.

31. Bai J, Brody JR, Kadkol SS and Pasternack GR: Tumor suppression and potentiation by manipulation of $\mathrm{pp} 32$ expression. Oncogene 20: 2153-2160, 2001.

32. Li W, Chen Z, Gong FR, Zong Y, Chen K, Li DM, Yin H, Duan WM, Miao Y, Tao M, et al: Growth of the pancreatic cancer cell line PANC-1 is inhibited by protein phosphatase 2A inhibitors through overactivation of the c-Jun N-terminal kinase pathway. Eur J Cancer 47: 2654-2664, 2011.

33. Schafer ZT, Parrish AB, Wright KM, Margolis SS, Marks JR, Deshmukh M and Kornbluth S: Enhanced sensitivity to cytochrome $c$-induced apoptosis mediated by PHAPI in breast cancer cells. Cancer Res 66: 2210-2218, 2006.

34. Kadkol SS, El Naga GA, Brody JR, Bai J, Gusev Y, Dooley WC and Pasternack GR: Expression of pp32 gene family members in breast cancer. Breast Cancer Res Treat 68: 65-73, 2001

35. Hill MM, Adrain C, Duriez PJ, Creagh EM and Martin SJ: Analysis of the composition, assembly kinetics and activity of native Apaf-1 apoptosomes. EMBO J 23: 2134-2145, 2004.
36. Kim HE, Jiang X, Du F and Wang X: PHAPI, CAS, and Hsp70 promote apoptosome formation by preventing Apaf-1 aggregation and enhancing nucleotide exchange on Apaf-1. Mol Cell 30: 239-247, 2008

37. Hoffarth S, Zitzer A, Wiewrodt R, Hähnel PS, Beyer V, Kreft A, Biesterfeld S and Schuler M: pp32/PHAPI determines the apoptosis response of non-small-cell lung cancer. Cell Death Differ 15: 161-170, 2008.

38. Brody JR, Witkiewicz A, Williams TK, Kadkol SS, Cozzitorto J, Durkan B, Pasternack GR and Yeo CJ: Reduction of pp32 expression in poorly differentiated pancreatic ductal adenocarcinomas and intraductal papillary mucinous neoplasms with moderate dysplasia. Mod Pathol 20: 1238-1244, 2007.

39. Shi H, Hood KA, Hayes MT and Stubbs RS: Proteomic analysis of advanced colorectal cancer by laser capture microdissection and two-dimensional difference gel electrophoresis. J Proteomics 75: 339-351, 2011.

40. Pekarčíková L, Knopfová L, Beneš P and Šmarda J: c-Myb regulates NOX1/p38 to control survival of colorectal carcinoma cells. Cell Signal 28: 924-936, 2016.

41. Li H, Huang K, Gao L, Wang L, Niu Y, Liu H, Wang Z, Wang L, Wang $\mathrm{G}$ and Wang J: TES inhibits colorectal cancer progression through activation of p38. Oncotarget 7: 45819-45836, 2016.

42. Fassetta M, D'Alessandro L, Coltella N, Di Renzo MF and Rasola A: Hepatocyte growth factor installs a survival platform for colorectal cancer cell invasive growth and overcomes p38 MAPK-mediated apoptosis. Cell Signal 18: 1967-1976, 2006.

43. Wang J, Huang F, Bai Z, Chi B, Wu J and Chen X: Curcumol inhibits growth and induces apoptosis of colorectal cancer LoVo cell line via IGF-1R and p38 MAPK pathway. Int J Mol Sci 16: 19851-19867, 2015

44. Yu LG, Packman LC, Weldon M, Hamlett J and Rhodes JM: Protein phosphatase $2 \mathrm{~A}$, a negative regulator of the ERK signaling pathway, is activated by tyrosine phosphorylation of putative HLA class II-associated protein I (PHAPI)/pp32 in response to the antiproliferative lectin, jacalin. J Biol Chem 279: 41377-41383, 2004.

45. Wang Z, Yang H, Tachado SD, Capó-Aponte JE, Bildin VN, Koziel H and Reinach PS: Phosphatase-mediated crosstalk control of ERK and p38 MAPK signaling in corneal epithelial cells. Invest Ophthalmol Vis Sci 47: 5267-5275, 2006.

46. Meng G, Wang W, Chai K, Yang S, Li F and Jiang K: Combination treatment with triptolide and hydroxycamptothecin synergistically enhances apoptosis in A549 lung adenocarcinoma cells through PP2A-regulated ERK, p38 MAPKs and Akt signaling pathways. Int J Oncol 46: 1007-1017, 2015.

47. Habrukowich C, Han DK, Le A, Rezaul K, Pan W, Ghosh M, Li Z, Dodge-Kafka K, Jiang X, Bittman R, et al: Sphingosine interaction with acidic leucine-rich nuclear phosphoprotein-32A (ANP32A) regulates PP2A activity and cyclooxygenase (COX)-2 expression in human endothelial cells. J Biol Chem 285: 26825-26831, 2010.

48. Da Silva M, Jaggers GK, Verstraeten SV, Erlejman AG, Fraga CG and Oteiza PI: Large procyanidins prevent bile-acid-induced oxidant production and membrane-initiated ERK1/2, p38, and Akt activation in Caco-2 cells. Free Radic Biol Med 52: 151-159, 2012.

49. Johnson SM, Gulhati P, Rampy BA, Han Y, Rychahou PG, Doan HQ, Weiss HL and Evers BM: Novel expression patterns of PI3K/Akt/mTOR signaling pathway components in colorectal cancer. J Am Coll Surg 210: 767-776, 776-768, 2010.

50. Chen B, Zeng X, He Y, Wang X, Liang Z, Liu J, Zhang P, Zhu H, $\mathrm{Xu} \mathrm{N}$ and Liang S: STC2 promotes the epithelial-mesenchymal transition of colorectal cancer cells through AKT-ERK signaling pathways. Oncotarget 7: 71400-71416, 2016

51. Baba Y, Nosho K, Shima K, Hayashi M, Meyerhardt JA, Chan AT, Giovannucci E, Fuchs CS and Ogino S: Phosphorylated AKT expression is associated with PIK3CA mutation, low stage, and favorable outcome in 717 colorectal cancers. Cancer 117: 1399-1408, 2011

52. Van Kanegan MJ, Adams DG, Wadzinski BE and Strack S: Distinct protein phosphatase $2 \mathrm{~A}$ heterotrimers modulate growth factor signaling to extracellular signal-regulated kinases and Akt. J Biol Chem 280: 36029-36036, 2005. 